\title{
HIGH DENSITY PIECEWISE SYNDETICITY OF PRODUCT SETS IN AMENABLE GROUPS
}

\author{
MAURO DI NASSO, ISAAC GOLDBRING, RENLING JIN, STEVEN LETH, \\ MARTINO LUPINI, AND KARL MAHLBURG
}

\begin{abstract}
M. Beiglböck, V. Bergelson, and A. Fish proved that if $G$ is a countable amenable group and $A$ and $B$ are subsets of $G$ with positive Banach density, then the product set $A B$ is piecewise syndetic. This means that there is a finite subset $E$ of $G$ such that $E A B$ is thick, that is, $E A B$ contains translates of any finite subset of $G$. When $G=\mathbb{Z}$, this was first proven by R. Jin. We prove a quantitative version of the aforementioned result by providing a lower bound on the density (with respect to a Følner sequence) of the set of witnesses to the thickness of $E A B$. When $G=\mathbb{Z}^{d}$, this result was first proven by the current set of authors using completely different techniques.
\end{abstract}

$\S 1$. Introduction. In the paper [6], R. Jin proved that if $A$ and $B$ are subsets of $\mathbb{Z}$ with positive Banach density, then $A+B$ is piecewise syndetic. This means that there is $m \in \mathbb{N}$ such that $A+B+[-m, m]$ is thick, i.e., it contains arbitrarily large intervals. Jin's result has since been extended in two different ways. First, using ergodic theory, M. Beiglböck, V. Bergelson, and A. Fish [1] established Jin's result for arbitrary countable amenable groups (with suitable notions of Banach density and piecewise syndeticity); in [5], M. Di Nasso and M. Lupini gave a simpler proof of the amenable group version of Jin's theorem using nonstandard analysis which works for arbitrary (not necessarily countable) amenable groups and also gives a bound on the size of the finite set needed to establish that the product set is thick. Second, in [4] the current set of authors established a "quantitative version" of Jin's theorem by proving that there is $m \in \mathbb{N}$ such that the set of witnesses to the thickness of $A+B+[-m, m]$ has upper density at least as large as the upper density of $A$. (We actually prove this result for subsets of $\mathbb{Z}^{d}$ for any $d$.) The goal of this article is to prove the quantitative version of the result of Beiglböck, Bergelson, and Fish.

In the following, we assume that $G$ is a countable amenable group ${ }^{1}$ : for every finite subset $E$ of $G$ and every $\varepsilon>0$, there exists a finite subset $L$ of $G$ such that, for every $x \in E$, we have $|x L \triangle L| \leq \varepsilon|L|$; such an $L$ is said to be $\epsilon$-Følner with respect

Received August 7, 2015.

2010 Mathematics Subject Classification. 11B13, 11B05, 03H05, 43 A07.

Key words and phrases. amenable groups, piecewise syndeticity, product sets.

${ }^{1}$ For those not familiar with amenable groups, let us mention in passing that the class of (countable) amenable groups is quite robust, e.g., contains all finite and abelian groups and is closed under subgroups, quotients, extensions, and direct limits. It follows, for example, that every countable virtually solvable group is amenable.

(C) 2016, Association for Symbolic Logic $0022-4812 / 16 / 8104-0017$ DOI:10.1017/js1.2015.75 
to $E$. It follows that $G$ is amenable if and only if it has a (left) Folner sequence, which is a sequence $\mathcal{S}=\left(S_{n}\right)$ of finite subsets of $G$ such that, for every $x \in G$, we have $\frac{\left|x S_{n} \triangle S_{n}\right|}{\left|S_{n}\right|} \rightarrow 0$ as $n \rightarrow+\infty$.

If $\mathcal{S}$ is a Følner sequence and $A \subseteq G$, we define the corresponding upper $\mathcal{S}$-density of $A$ to be

$$
\bar{d}_{\mathcal{S}}(A):=\limsup _{n \rightarrow+\infty} \frac{\left|A \cap S_{n}\right|}{\left|S_{n}\right|} .
$$

For example, note that if $G=\mathbb{Z}^{d}$ and $\mathcal{S}=\left(S_{n}\right)$ where $S_{n}=[-n, n]^{d}$, then $\bar{d}_{\mathcal{S}}$ is the usual notion of upper density for subsets of $\mathbb{Z}^{d}$.

Following [1], we define the (left) Banach density of $A, \mathrm{BD}(A)$, to be the supremum of $\bar{d}_{\mathcal{S}}(A)$ where $\mathcal{S}$ ranges over all Følner sequences of $G$. One can verify (see [1]) that this notion of Banach density agrees with the usual notion of Banach density when $G=\mathbb{Z}^{d}$.

Recall that a subset $A$ of $G$ is thick if for every finite subset $L$ of $G$ there exists a right translate $L x$ of $L$ contained in $A$. A subset $A$ of $G$ is piecewise syndetic if $F A$ is thick for some finite subset $F$ of $G$.

Definition 1.1. Suppose that $G$ is a countable discrete group, $\mathcal{S}$ is a Følner sequence for $G, A$ is a subset of $G$, and $\alpha>0$. We say that $A$ is

- upper $\mathcal{S}$-thick of level $\alpha$ if for every finite subset $L$ of $G$, the set $\{x \in G: L x \subseteq A\}$ has upper $\mathcal{S}$-density at least $\alpha$;

- upper $\mathcal{S}$-syndetic of level $\alpha$ if there exists a finite subset $F$ of $G$ such that $F A$ is upper $\mathcal{S}$-thick of level $\alpha$.

The following is the first main result of this paper:

Theorem 1.2. Suppose that $G$ is a countable amenable group and $\mathcal{S}$ is a Folner sequence for $G$. If $A$ and $B$ are subsets of $G$ such that $\bar{d}_{\mathcal{S}}(A)=\alpha>0$ and $\operatorname{BD}(B)>0$, then $B A$ is upper $\mathcal{S}$-syndetic of level $\alpha$.

When $G=\mathbb{Z}^{d}$ and $\mathcal{S}=\left(S_{n}\right)$ where $S_{n}=[-n, n]^{d}$, we recover [4, Theorem 14]. We also recover [4, Theorem 18], which states (in the current terminology) that if $A, B \subseteq \mathbb{Z}^{d}$ are such that $\underline{d}(A)=\alpha>0$ and $\operatorname{BD}(B)>0$, then $A+B$ is $\mathcal{S}^{\prime}$-syndetic of level $\alpha$ for any subsequence $\mathcal{S}^{\prime}$ of the aforementioned $\mathcal{S}$.

If $\mathcal{S}$ is a Følner sequence for $G$, then the notions of lower $\mathcal{S}$-density $\underline{d}_{S}$, lower $\mathcal{S}$-thick, and lower $\mathcal{S}$-syndetic can be defined in the obvious ways. In [4, Theorem 19], the current set of authors proved that if $A, B \subseteq \mathbb{Z}^{d}$ are such that $\underline{d}_{\mathcal{S}}(A)=\alpha>0$ and $\mathrm{BD}(B)>0$ (where $\mathcal{S}$ is the usual Følner sequence for $\mathbb{Z}^{d}$ as above), then for any $\epsilon>0, A+B$ is lower $\mathcal{S}$-syndetic of level $\alpha-\epsilon$. (An example is also given to show that, under the previous hypotheses, $A+B$ need not be lower $\mathcal{S}$-syndetic of level $\alpha$.) In a previous version of this paper, we asked whether or not the amenable group analog of [4, Theorem 19] was true. Using ergodic-theoretic methods, Michael Björklund [2] settled this question in the affirmative. Shortly after, we realized that our techniques readily established the same result and we include our proof here.

In their proof of the amenable group version of Jin's theorem, the authors of [5] give a bound on the size of a finite set needed to witness piecewise syndeticity: if $G$ is a countable amenable group and $A$ and $B$ are subsets of $G$ of Banach densities 
$\alpha$ and $\beta$ respectively, then there is a finite subset $E$ of $G$ with $|E| \leq \frac{1}{\alpha \beta}$ such that $E A B$ is thick. In Section 3, we improve upon this theorem in two ways: we slightly improve the bound on $|E|$ from $\frac{1}{\alpha \beta}$ to $\frac{1}{\alpha \beta}-\frac{1}{\alpha}+1$ and we show that, if $\mathcal{S}$ is any Følner sequence such that $\bar{d}_{\mathcal{S}}(A)>0$, then $E A B$ is $\mathcal{S}$-thick of level $s$ for some $s>0$.

1.1. Notions from nonstandard analysis. We use nonstandard analysis to prove our main results. An introduction to nonstandard analysis with an eye towards applications to combinatorics can be found in [7]. Here, we just fix notation.

If $r, s$ are finite hyperreal numbers, we write $r \lesssim s$ to mean $\operatorname{st}(r) \leq \operatorname{st}(s)$ and we write $s \approx r$ to mean $\operatorname{st}(r)=\operatorname{st}(s)$.

If $X$ is a hyperfinite subset of ${ }^{*} G$, we denote by $\mu_{X}$ the corresponding Loeb measure, which is a probability measure extending the finitely additive probability measure defined on the algebra of internal subsets of $X$ given by $\mu_{X}(A):=\operatorname{st}\left(\frac{|A|}{|X|}\right)$. If, moreover, $Y \subseteq{ }^{*} G$ is internal, we abuse notation and write $\mu_{X}(Y)$ for $\mu_{X}(X \cap Y)$.

Suppose that $\mathcal{S}=\left(S_{n}\right)$ is a Følner sequence for $G$. For $v$ an infinite hypernatural number, we denote by $S_{v}$ the value at $v$ of the nonstandard extension of $\mathcal{S}$. It follows readily from the definition that $\bar{d}_{\mathcal{S}}(A)$ is the maximum of $\mu_{S_{v}}\left({ }^{*} A\right)$ as $v$ ranges over all infinite hypernatural numbers.

The following definition is central.

Definition 1.3. Suppose that $G$ is a group and $X \subseteq{ }^{*} G$ is hyperfinite. We say that $X$ is a Folner approximation of $G$ if $|g X \triangle X| /|X|$ is infinitesimal for every $g \in G$.

It follows immediately from the nonstandard characterization of limit that if $G$ is a countable amenable group and $\left(S_{n}\right)$ is a Følner sequence for $G$, then $S_{v}$ is a Følner approximation of $G$ for every $v>\mathbb{N}^{2}$

$\S 2$. High density piecewise syndeticity. In this section, $G$ denotes a countable amenable group. We begin with a combinatorial lemma.

Lemma 2.1. Suppose that $\mathcal{S}=\left(S_{n}\right)$ is a Følner sequence for $G, Y$ a Følner approximation for $G$, and $A \subseteq G$.

(1) If $\bar{d}_{\mathcal{S}}(A) \geq \alpha$, then there is $v>\mathbb{N}$ such that

$$
\frac{\left|{ }^{*} A \cap S_{v}\right|}{\left|S_{v}\right|} \gtrsim \alpha \text { and } \frac{1}{\left|S_{v}\right|} \sum_{x \in S_{v}} \frac{\left|x\left({ }^{*} A \cap S_{v}\right)^{-1} \cap Y\right|}{|Y|} \gtrsim \alpha .
$$

(2) If $\underline{d}_{\mathcal{S}}(A)>\alpha$, then there is $v_{0}>\mathbb{N}$ such that $(\dagger)$ holds for all $v \geq v_{0}$.

Proof. For (1), first apply transfer to the statement "for every finite subset $E$ of $G$ and every natural number $k$, there exists $n \geq k$ such that

$$
\frac{1}{\left|S_{n}\right|}\left|A \cap S_{n}\right|>\alpha-2^{-k} \text { and } \frac{1}{\left|S_{n}\right|} \sum_{x \in E}\left|x^{-1} S_{n} \triangle S_{n}\right|<2^{-k} . "
$$

\footnotetext{
${ }^{2}$ Conversely, if $G$ admits a Følner approximation $X$, then $G$ is amenable. Indeed, given finite $E \subseteq G$ and $\epsilon>0$, observe that $X$ is $\epsilon$-Følner with respect to $E$, whence an actual (standard) $\epsilon$-Følner set for $E$ exists by downward transfer.
} 
Fix $K>\mathbb{N}$ and let $v$ be the result of applying the transferred statement to $Y$ and $K$. Set $C={ }^{*} A \cap S_{v}$ and let $\chi_{C}$ denote the characteristic function of $C$. We have

$$
\begin{aligned}
\frac{1}{\left|S_{v}\right|} \sum_{x \in S_{v}} \frac{\left|x C^{-1} \cap Y\right|}{|Y|} & =\frac{1}{\left|S_{v}\right|} \sum_{x \in S_{v}} \frac{1}{|Y|} \sum_{y \in Y} \chi_{C}\left(y^{-1} x\right) \\
& =\frac{1}{|Y|} \sum_{y \in Y} \frac{\left|C \cap y^{-1} S_{v}\right|}{\left|S_{v}\right|} \\
& \geq \frac{|C|}{\left|S_{v}\right|}-\sum_{y \in Y} \frac{\left|y^{-1} S_{v} \triangle S_{v}\right|}{\left|S_{v}\right|} \\
& \approx \alpha .
\end{aligned}
$$

For (2), apply transfer to the statement "for every finite subset $E$ of $G$ and every natural number $k$, there exists $n_{0} \geq k$ such that, for all $n \geq n_{0}$,

$$
\frac{1}{\left|S_{n}\right|}\left|A \cap S_{n}\right|>\alpha-2^{-n_{0}} \quad \text { and } \quad \frac{1}{\left|S_{n}\right|} \sum_{x \in E}\left|x^{-1} S_{n} \triangle S_{n}\right|<2^{-n_{0}} . "
$$

Once again, fix $K>\mathbb{N}$ and let $v_{0}$ be the result of applying the transferred statement to $Y$ and $K$. As above, this $v_{0}$ is as desired.

Suppose that $B \subseteq G$ is such that $\operatorname{BD}(B) \geq \beta$. It is a routine saturation argument to see that there is then a Følner approximation $Y$ of $G$ such that $\frac{\left.\right|^{*} B \cap Y \mid}{|Y|} \gtrsim \beta$. It will become important to demand an extra condition on such a Følner approximation.

Definition 2.2. Suppose that $B \subseteq G$ is such that $\mathrm{BD}(B) \geq \beta$. We say a Følner approximation $Y$ of $G$ is good for $B$ if

$$
\frac{\left|{ }^{*} B \cap Y\right|}{|Y|} \gtrsim \beta
$$

and, for any standard $\varepsilon>0$, there exists a finite subset $F$ of $G$ such that

$$
\frac{|*(F B) \cap Y|}{|Y|}>1-\varepsilon .
$$

The following fact is central to our arguments and its proof is contained in the proof of [3, Lemma 4.6].

FACT 2.3. If $B \subseteq G$ is such that $\mathrm{BD}(B)>0$, then there is a Følner approximation $Y$ of $G$ that is good for $B$.

We are now ready to prove the main results of this section.

THEOREM 2.4. Suppose that $G$ is a countable amenable group, $\mathcal{S}=\left(S_{n}\right)$ a Folner sequence for $G$, and $A, B \subseteq G$. If $\bar{d}_{\mathcal{S}}(A) \geq \alpha$ and $\mathrm{BD}(B)>0$, then $B A$ is upper $\mathcal{S}$-syndetic of level $\alpha$.

Proof. Let $Y$ be a Følner approximation for $G$ that is good for $B$. Let $v$ be as in part (1) of Lemma 2.1 applied to $Y$ and $A$. Once again, set $C:={ }^{*} A \cap S_{v}$. Consider the $\mu_{S_{v}}$-measurable function

$$
f(x)=\operatorname{st}\left(\frac{\left|x C^{-1} \cap Y\right|}{|Y|}\right) .
$$


Observe that

$$
\int_{S_{v}} f d \mu_{S_{v}}=\operatorname{st}\left(\frac{1}{\left|S_{v}\right|} \sum_{x \in S_{v}} \frac{\left|x C^{-1} \cap Y\right|}{|Y|}\right) \geq \alpha,
$$

whence there is some standard $r>0$ such that $\mu_{S_{v}}\left(\left\{x \in S_{v}: f(x) \geq 2 r\right\}\right) \geq \alpha$. Setting $\Gamma=\left\{x \in S_{v}: \frac{\left|x C^{-1} \cap Y\right|}{|Y|} \geq r\right\}$, we have that $\mu_{S_{v}}(\Gamma) \geq \alpha$. Since $Y$ is good for $B$, we may take a finite subset $F$ of $G$ such that

$$
\frac{|*(F B) \cap Y|}{|Y|}>1-\frac{r}{2}
$$

Fix $g \in G$. Since $Y$ is a Følner approximation for $G$, we have that

$$
\frac{\left|{ }^{*}(g F B) \cap Y\right|}{|Y|}=\frac{\left|{ }^{*}(F B) \cap g^{-1} Y\right|}{|Y|} \approx \frac{|*(F B) \cap Y|}{|Y|},
$$

whence $\frac{{ }^{*}(g F B) \cap Y \mid}{|Y|}>1-r$. Thus, for any $x \in \Gamma$, we have that $x C^{-1} \cap *(g F B) \neq \emptyset$. In particular, if $L$ is a finite subset of $G$, then $\Gamma \subseteq \subseteq^{*}\left(\bigcap_{g \in L} g F B A\right)$. Therefore

$$
\bar{d}_{\mathcal{S}}\left(\bigcap_{g \in L} g F B A\right) \geq \mu_{S_{v}}\left(*\left(\bigcap_{g \in L} g F B A\right)\right) \geq \mu_{S_{v}}(\Gamma) \geq \alpha .
$$

It follows that $B A$ is upper $\mathcal{S}$-syndetic of level $\alpha$.

As mentioned in the introduction, Theorems 14 and 18 of [4] are immediate consequences of Theorem 2.4, after observing that the sequence of sets $[-n, n]^{d}$ as well as any of its subsequences is a Følner sequence for $\mathbb{Z}^{d}$. Example 15 of [4] shows that the conclusion in Theorem 2.4 is optimal, even when $G$ is the additive group of integers and $\mathcal{S}$ is the Følner sequence of intervals $[1, n]$.

Theorem 2.5. Suppose that $G$ is a countable amenable group, $\mathcal{S}=\left(S_{n}\right)$ a Følner sequence for $G$, and $A, B \subseteq G$. If $\underline{d}_{\mathcal{S}}(A) \geq \alpha$ and $\mathrm{BD}(B)>0$, then $B A$ is lower $\mathcal{S}$-thick of level $\alpha-\varepsilon$ for every $\varepsilon>0$.

Proof. Without loss of generality, we may suppose that $\underline{d}_{\mathcal{S}}(A)>\alpha$. Fix a Følner approximation $Y$ for $G$ that is good for $B$ and $v_{0}>\mathbb{N}$ as in part (2) of Lemma 2.1 applied to $Y$ and $A$. Fix $v \geq v_{0}$ and standard $\varepsilon>0$ with $\varepsilon<\alpha$. Set

$$
\Gamma_{v}=\left\{x \in S_{v}: \frac{\left|x C^{-1} \cap Y\right|}{|Y|} \geq \varepsilon\right\}
$$

and observe that $\frac{\left|\Gamma_{v}\right|}{\left|S_{v}\right|}>\alpha-\varepsilon$. Since $Y$ is $\operatorname{good}$ for $B$, we may fix a finite subset $F$ of $G$ such that

$$
\frac{|*(F B) \cap Y|}{|Y|}>1-\frac{\varepsilon}{2} .
$$

Fix $g \in G$. Since $Y$ is a Følner approximation of $G$, arguing as in the proof of the previous theorem, we conclude that

$$
\frac{\left|{ }^{*}(g F B) \cap Y\right|}{|Y|}>1-\varepsilon
$$


Fix $L \subseteq G$ finite. Once again, it follows that $\Gamma_{v} \subseteq *\left(\bigcap_{g \in L} g F B A\right)$ whence

$$
\frac{\left|*\left(\bigcap_{g \in L} g F B A\right) \cap S_{v}\right|}{\left|S_{v}\right|} \geq \frac{\left|\Gamma_{v}\right|}{\left|S_{v}\right|}>\alpha-\varepsilon .
$$

Since the previous inequality held for every $v \geq v_{0}$, by transfer we can conclude that

$$
\underline{d}_{\mathcal{S}}\left(\bigcap_{g \in L} g F B A\right) \geq \alpha-\varepsilon .
$$

It follows that $B A$ is lower $\mathcal{S}$-syndetic of level $\alpha-\epsilon$.

As mentioned in the introduction, Theorem 2.5 is a generalization of [4, Theorem 19] and was first proven by M. Björklund using ergodic-theoretic methods.

§3. A bound on the number of translates. The following theorem is a refinement of [5, Corollary 3.4]. In particular, we improve the bound on the number of translates, and also obtain an estimate on the $\mathcal{S}$-density of translates that witness the thickness of $E B A$.

THEOREM 3.1. Suppose that $G$ is a countable amenable group, $\mathcal{S}=\left(S_{n}\right)$ a Følner sequence for $G$, and $A, B \subseteq G$. If $d_{\mathcal{S}}(A) \geq \alpha$ and $\mathrm{BD}(B) \geq \beta$, then there exists $s>0$ and a finite subset $E \subseteq G$ such that $|\bar{E}| \leq \frac{1}{\alpha \beta}-\frac{1}{\alpha}+1$ and $E B A$ is $\mathcal{S}$-thick of level $s$.

Proof. Reasoning as in the proof of Theorem 2.4, one can prove that there exist $v>\mathbb{N}$, a Følner approximation $Y$ of $G$, a standard $r>0$, and an infinitesimal $\eta \in{ }^{*} \mathbb{R}_{+}$such that, if $C={ }^{*} A \cap S_{v}, D={ }^{*} B \cap Y$, and

$$
\Gamma:=\left\{x \in S_{v}: \frac{1}{|Y|}\left|x C^{-1} \cap D\right| \geq \alpha \beta-\eta\right\},
$$

then $|\Gamma| \geq r\left|S_{v}\right|$. Fix a family $\left(p_{g}\right)_{g \in G}$ of strictly positive standard real numbers such that $\sum_{g \in G} p_{g} \leq \frac{1}{2}$.

We now define a sequence of subsets $\left(H_{n}\right)$ of $G$ and a sequence $\left(s_{n}\right)$ from $G$. Define

$$
H_{0}:=\left\{g \in G: \frac{1}{|\Gamma|}\left|\left\{x \in \Gamma: g x \notin^{*}(B A)\right\}\right|>p_{g}\right\} .
$$

If $H_{n}$ has been defined and is nonempty, let $s_{n}$ be any element of $H_{n}$ and set

$$
H_{n+1}:=\left\{g \in G: \frac{1}{|\Gamma|}\left|\left\{x \in \Gamma: g x \notin^{*}\left(\left\{s_{0}, \ldots, s_{n}\right\} B A\right)\right\}\right|>p_{g}\right\} .
$$

If $H_{n}=\varnothing$ then we set $H_{n+1}=\varnothing$.

We claim $H_{n}=\emptyset$ for $n>\left\lfloor\frac{1}{\alpha \beta}-\frac{1}{\alpha}\right\rfloor$. Towards this end, suppose $H_{n} \neq \varnothing$. For $0 \leq k \leq n$, take $\gamma_{k} \in \Gamma$ such that

$$
s_{k} \gamma_{k} \notin *\left(\left\{s_{0}, \ldots, s_{k-1}\right\} B A\right) .
$$

Observe that the sets $s_{0} D, s_{1}\left(\gamma_{1} C^{-1} \cap D\right), \ldots, s_{n}\left(\gamma_{n} C^{-1} \cap D\right)$ are pairwise disjoint. In fact, if

$$
s_{i} D \cap s_{j} \gamma_{j} C^{-1} \neq \varnothing
$$


for $0 \leq i<j \leq n$, then

$$
s_{j} \gamma_{j} \in s_{i} D C \subseteq \subseteq^{*}\left(\left\{s_{0}, \ldots, s_{j-1}\right\} B A\right),
$$

contradicting the choice of $\gamma_{j}$. Therefore we have that

$$
\begin{aligned}
1 & \gtrsim \frac{1}{|Y|}\left|s_{0} D \cup s_{1}\left(\gamma_{1} C^{-1} \cap D\right) \cup \cdots \cup s_{n}\left(\gamma_{n} C^{-1} \cap D\right)\right| \\
& \geq \frac{1}{|Y|}\left(|D|+\sum_{i=1}^{n}\left|\gamma_{i} C^{-1} \cap D\right|\right) \\
& \geq \beta+\alpha \beta n .
\end{aligned}
$$

It follows that $n \leq\left\lfloor\frac{1}{\alpha \beta}-\frac{1}{\alpha}\right\rfloor$.

Take the least $n$ such that $H_{n}=\emptyset$. Note that $n \leq\left\lfloor\frac{1}{\alpha \beta}-\frac{1}{\alpha}\right\rfloor+1$. If $n=0$ then $B A$ is already $\mathcal{S}$-thick of level $r$, and there is nothing to prove. Let us assume that $n \geq 1$, and set $E=\left\{s_{0}, \ldots, s_{n-1}\right\}$. It follows that, for every $g \in G$, we have that

$$
\frac{1}{|\Gamma|}\left|\left\{x \in \Gamma: g x \in^{*}(E B A)\right\}\right| \geq 1-p_{g} .
$$

Suppose that $L$ is a finite subset of $G$. Then

$$
\frac{1}{|\Gamma|}\left|\left\{x \in \Gamma: L x \subseteq{ }^{*}(E B A)\right\}\right| \geq 1-\sum_{g \in L} p_{g} \geq 1-\sum_{g \in G} p_{g} \geq \frac{1}{2} .
$$

Therefore

$$
\frac{1}{\left|S_{v}\right|}\left|\left\{x \in S_{v}: L x \subseteq{ }^{*}(E B A)\right\}\right| \geq \frac{r}{2} .
$$

This shows that $E B A$ is $\mathcal{S}$-thick of level $\frac{r}{2}$.

With a similar argument using Markov's inequality [8, Lemma 1.3.15], one can also prove the following result. We omit the details.

THEOREM 3.2. Suppose that $G$ is a countable amenable group, $\mathcal{S}=\left(S_{n}\right)$ a Folner sequence for $G$, and $A, B \subseteq G$. If $d_{\mathcal{S}}(A) \geq \alpha$ and $\mathrm{BD}(B) \geq \beta$, then for every $\gamma \in(0, \alpha \beta]$ there exists $E \subseteq G$ such that $|E| \leq \frac{1-\beta}{\gamma}+1$ and $E B A$ is $\mathcal{S}$-syndetic of level $\frac{\alpha \beta-\gamma}{1-\gamma}$.

§4. Acknowledgements. This work was initiated during a week-long meeting at the American Institute for Mathematics on August 4-8, 2014 as part of the SQuaRE (Structured Quartet Research Ensemble) project "Nonstandard Methods in Number Theory." The authors would like to thank the Institute for the opportunity and for the Institute's hospitality during their stay. The authors would also like to thank the anonymous referee of a previous version of this paper for helpful remarks that allowed us to sharpen our results.

The authors were supported in part by the American Institute of Mathematics through its SQuaREs program. I. Goldbring was partially supported by NSF CAREER grant DMS-1349399. M. Lupini was supported by the York University Susan Mann Dissertation Scholarship and by the ERC Starting grant no. 259527 of Goulnara Arzhantseva. K. Mahlburg was supported by NSF Grant DMS-1201435. 
M. Di Nasso was supported by MIUR-PRIN Grant "Models and sets" and by the University of Pisa Grant PRA 2015/005.

\section{REFERENCES}

[1] M. Beiglböck, V. Bergelson, and A. Fish, Sumset phenomenon in countable amenable groups. Advances in Mathematics, vol. 223 (2010), no. 2, pp. 416-432.

[2] M. BJöRKLUND, personal communication.

[3] M. Di Nasso, I. Goldbring, R. Jin, S. Leth, M. Lupini, and K. Mahlburg, On a sumset conjecture of Erdös. Canadian Journal of Mathematics, 2014, pp. 1-17.

[4] — - High density piecewise syndeticity of sumsets. Advances in Mathematics, vol. 278 (2015), pp. 1-33.

[5] M. Di NASso and M. LuPINI, Nonstandard analysis and the sumset phenomenon in arbitrary amenable groups. Illinois Journal of Mathematics, vol. 58 (2014), no. 1, pp. 11-25.

[6] R. JIN, The sumset phenomenon. Proceedings of the American Mathematical Society, vol. 130 (2002), no. 3, pp. 855-861.

[7] - Introduction of nonstandard methods for number theorists, Integers. Electronic Journal of Combinatorial Number Theory, vol. 8 (2008), no. 2.

[8] T. TAO, An Introduction to Measure Theory, Graduate Studies in Mathematics, vol. 126, American Mathematical Society, Providence, RI, 2011.

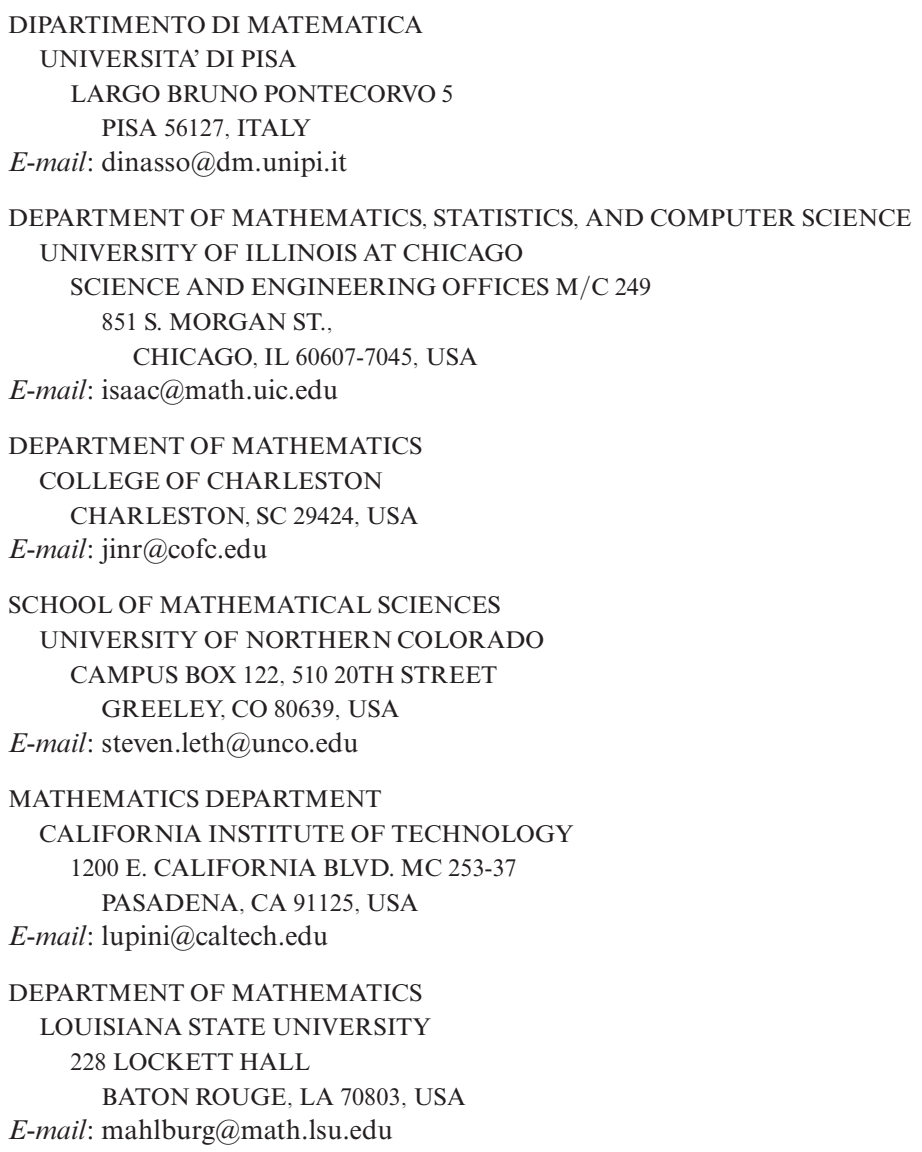

E-mail: mahlburg@math.lsu.edu 